\title{
Quantum Cascade Laser Photoacoustic Spectroscopy Applied to Rice Flour Analysis
}

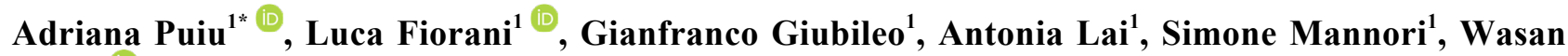 \\ Saleh $^{2}$
}

${ }^{1}$ ENEA, Nuclear Fusion and Safety Technologies Department, Via E. Fermi 45, 00044 Frascati, Italy

${ }^{2}$ ENEA guest, Department of Physics, College of Science, University of Baghdad, Baghdad, Iraq

E-mail: adriana.puiu@enea.it

Received: 22 May 2021; Revised: 25 August 2021; Accepted: 3 September 2021

\begin{abstract}
In the present work we applied laser photoacoustic spectroscopy (LPAS) to the analysis of food samples. In particular, we analyzed samples of different rice flours (standard and commercial ones). For this purpose, a laboratory system based on quantum cascade laser (QCL) has been developed and characterized. After that, the LPAS spectra of all the rice samples were collected with a standard error of less than $2 \%$ of the measured value and a background signal negligible compared to the sample signals. All the experimental LPAS spectra resulted to be rich in spectral features showing clear differences between each other. The experimental spectra were then analyzed by comparison with the FTIR transition frequency recorded in our laboratory to consent a proper assignment. Finally, to put in evidence the small differences among the various rice types, the Principal Component Analysis (PCA) was applied to the recorded LPAS spectra highlighting five different groups corresponding to the five types of samples. In conclusion, the present work demonstrated the discriminating capability of LPAS technique in the case of different types of rice flour samples.
\end{abstract}

Keywords: rice flour, fast detection, laser spectroscopy, photoacoustic technique, chemometrics

\section{Introduction}

The development of early and non-destructive diagnostic systems with high capability for analysis, discrimination, sensitivity, and fast response time is a general request in all the fields affecting people's health: biomedicine, homeland security, environment, and water monitoring, as well as in the agro-food sector. In the last two decades, the applicability of optical and spectroscopic techniques has proved its effectiveness also in post-harvesting, along the distribution chain, due to its non-invasive characteristics and continuous monitoring of the components that can be released and which affect the nutritional value of food [1]. The use of these technologies, however, is still confined in laboratories or large industries, so its technological development for the simplification of the system that makes it manageable and easy to use along the supply chain, is one of the objectives of recent years. There are already on the market a few examples of devices that are able to detect in a non-destructive way the quality of the fruit [2-3]. Following the expansion of markets from local to global, the supply chains have become more complex and this has opened the door to food fraud on a larger scale. Thus, manufacturers and consumers are demanding not only increased legislative protection but also fast and efficient control methods. The research presented in this paper is intended to be the basis for the manufacturing of

Copyright (C2021 Adriana Puiu, et al.

DOI: https://doi.org/10.37256/fse.222021956

This is an open-access article distributed under a CC BY license

(Creative Commons Attribution 4.0 International License)

https://creativecommons.org/licenses/by/4.0/ 
a compact and sensitive prototype for fast characterization of foodstuff by means of laser photoacoustic spectroscopy (LPAS). This device will be interfaced with a database of food types which are most subject to adulteration, in order to compare the sample under test with the spectroscopic fingerprints of standard materials. Rapid screening methods are particularly important to the industry, where an answer is needed within seconds and, of course, supporting reference materials and spectral libraries are indispensable. The concept is to detect food quality by examining a fingerprint for a given ingredient to check that it matches the known fingerprint. The advantage of this approach is that it can detect both known and unknown adulterants [4]. This is a powerful approach, although its development is challenging.

Laboratory techniques generally used to characterize food and to detect adulterants are liquid chromatography/ mass spectrometry (LC/MS), gas chromatography/mass spectrometry (GC/MS) that are expensive, time-consuming, and request specialized personnel. In some cases, rapid techniques such as Fourier transform infrared (FT-IR) spectral analysis that picks up almost all the organic compounds and also some inorganics, Raman spectroscopy, matrix-assisted laser desorption/ionization mass spectrometry, nuclear magnetic resonance (NMR), and laser spectroscopy are used. All these techniques are non-targeted analytical methods but they have the great advantage to give an immediate alert in case of foodstuff out of standard parameters. Moreover, laser spectroscopy provides a higher resolution given by the very narrow bandwidth of the laser source (in our case $0.001 \mathrm{~cm}^{-1}$ ) with respect to the spectroscopy apparatus using thermal light sources (i.e. globars), Nernst lamps, coils of chrome-nickel alloy, or high-pressure mercury lamps, etc. A previous spectroscopy apparatus based on a continuous wave $(\mathrm{CW}) \mathrm{CO}_{2}$ laser already proved to be a useful tool for some issues such as contamination of seafood, adulteration of sugar, melamine presence in powder milk, early detection of orange fruit infestation with pathogens, etc. [5-8]. The reported quantum cascade laser (QCL) based system was carefully characterized by recording the emitted power profile of the laser source over the entire tunable range, by identifying the photoacoustic (PA) cell resonance frequency, and finally by checking the linearity of the PA response when varying the laser power.

In the present paper, we report on the LPAS measurements of different commercial rice flour samples in order to compare differences or similarities among these testers. As a control sample, the standard provided by the National Institute of Standards and Technology (NIST) [9] was also measured. Rice (Oryza sativa L.) is the staple food of almost half the world's population. It is the predominant dietary energy source for many developing countries especially in Asia, South America, and Africa, providing 20\% of the world's dietary energy supply [10]. In the European Union (EU), Italy and Spain are the largest rice producers [11]. Approximately two-thirds of Europe's rice is produced in Italy and approximately two thirds of the national production is exported both towards the EU countries (two-thirds of the exported amount) and other countries in the Mediterranean area and Eastern Europe. Thus, rice is one of the most economically important cereal crops in Italy in view of foreign exchange [12]. Consequently, it has become a potential target for many unscrupulous importers for mixing with low-grade, low-cost products and low nutritious adulterants to fetch profits with the least efforts. To protect the interests of importing countries and consumers, several methods have been employed over the last few decades for unambiguous discrimination of cultivars, accurate quantification of the adulterants, and for determination of cultivated geographical area [13] but it is the first time to our knowledge when LPAS spectroscopy is employed for the rice flour analysis. Results on the analysis and identification of five varieties of rice flour by a compact and sensitive prototype based on the LPAS technique combined with chemometric analysis will be reported.

\section{Material and methods}

\subsection{Rice samples}

Commercial Basmati and Carnaroli cultivars (cv) of rice (Oryza sativa L.) were analyzed for this study. Basmati was purchased from 2 different companies (Basmati $\mathrm{C}$ and Basmati E) while the commercial Carnaroli was compared with a certified one (Metrofood) [14]. The rice is subdivided into three species: short-bodied Japonica (e.g. Carnaroli, Arborio, etc.), Indica with long and thin grains (Basmati, Long thai), and Javanica, little known, with wide and flat grains, widespread in the island of Java, hence the name. The NIST standard is rice flour obtained from $100 \%$ long grain river rice grown in Arkansas.

In order to compare our rice samples to the standard, provided by NIST on flour form, all samples were grinded 
for 15 minutes at room temperature by using a Spectromill Ball Pestle Impact Grinder produced by Chemplex Inc. The rice samples powders were produced by a simple grinding operation without any extraction, purification or other manipulation process. To perform the LPAS measurement, a small amount (1 mg) of the obtained powder was inserted into the sample holder located on the bottom of the PA cell, the cell was closed and the laser was fired. During the measurement process the laser beam interacts directly with the powder sample inside the PA cell giving rise to the PA signal as described later on. Before insert a new sample, the PA cell and the holder sample were thoroughly cleaned with pure isopropyl alcohol and dried by air.

\section{$2.2 F T-I R / A T R$}

Fourier Transform Infrared spectroscopy (FT-IR) is a spectroscopic absorption technique used in the field of analytical chemistry and characterization of materials for the study of chemical bonds. Our FTIR apparatus was equipped with the ATR (Attenuated Total Reflectance) option. The technique ATR exploits the reflection of the ray that passes through the sample. In this technique the sample is placed in close contact with an optical element of internal reflection (ATR crystal) with a high index of refraction. The accessory for the ATR is equipped with a diamond crystal. The IR beam emitted from the source, before reaching the sample, passes first through this element: when the angle of incidence is greater than the critical angle, the phenomenon known as total reflection takes place. This reflected ray, arriving on the surface of the sample that constitutes the interface, can penetrate up to a thickness of $2 \mu \mathrm{m}$ of the less refractive material. In this way it forms an evanescent wave, which as a result of the radiation absorption from the sample will produce an attenuated ray; in this way it is possible to record the ATR spectrum.

In the present work, to perform the ATR analysis, IRAffinity-1S system (by Shimadzu) was used, equipped with a high-energy ceramic light source and a very sensitive, temperature-controlled pyroelectric detector in Deuterated Lanthanum $\alpha$ Alanine doped TriGlycine Sulphate (DLATGS). The apparatus has been set to operate in the spectral range 550-3800 $\mathrm{cm}^{-1}$ with a spectral resolution of $2 \mathrm{~cm}^{-1}$.

\subsection{LPAS technique}

\subsubsection{Set-up and measurement procedure}

The measurement technique is based on the generation of the photoacoustic effect [15] induced by the interaction between the rice flour sample hold inside the photoacoustic (PA) cell and the laser radiation beam that is power modulated at an audible frequency (in our case $215 \mathrm{~Hz}$, determined as described in the following of this paper). Inside the PA cell, this interaction generates an acoustic pressure wave proportional in intensity to the sample absorption coefficient and is detected by a sensitive microphone which converts the sound to an electric signal. The microphone electric signal intensity does represent the PA experimental data. The block diagram of the instrument is given in Figure 1.

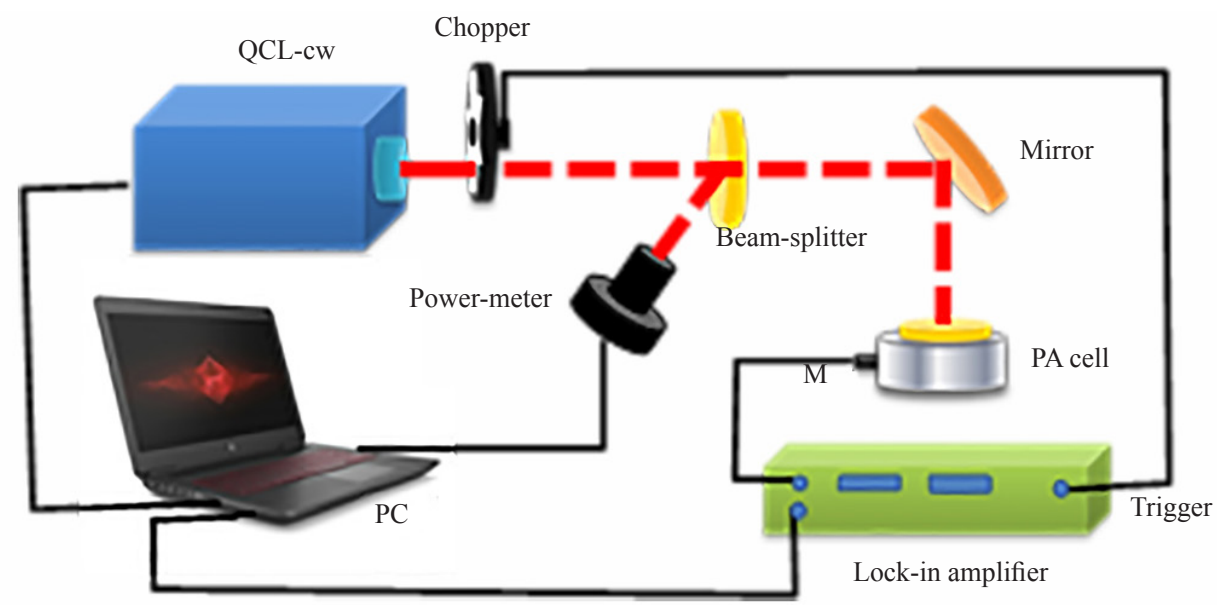

Figure 1. Block diagram of the QCL based LPAS system. M: high sensitivity miniaturized microphone 
Our LPAS optical apparatus is equipped with: a quantum cascade laser-QCL (Model MIRcat-1100 by Daylight Solutions) having maximum emission power of about $40 \mathrm{~mW}$ continuous wave (when setting the laser current at 1100 $\mathrm{mA})$, linewidth $<100 \mathrm{MHz}\left(\sim 0.0033 \mathrm{~cm}^{-1}\right)$, tunable in the range 8.4-11 $\mu \mathrm{m}$; a one-channel power meter (Maestro Model with XPL12-1S-H2-DO probe by Gentec); a photoacoustic cell designed and realized in our laboratory (internal volume about $3 \mathrm{~cm}^{-3}$ ); and a lock-in amplifier (Model SR 830 by Stanford Research Systems). The emission from the QCL is modulated with a square wave with $50 \%$ duty cycle by a mechanical chopper (Model SR 540 by Stanford Research Systems $)$ at the resonance frequency $(218 \mathrm{~Hz})$ of the photoacoustic cell. The PA signal generated inside the cell is detected by a miniaturized sensitive microphone (dimensions in mm $5.6 \times 4.1 \times 2.2$ ) by Knowles (Model EK-23024) and sent to the lock-in amplifier, which communicates with the PC via USB. Data are acquired through a dedicated software for control and acquisition developed in our laboratory in the Matlab environment.

To obtain a spectrum through the LPAS method, the sample holder containing a small amount (1 mg) of the rice powder understudy was inserted into the PA cell previously cleaned, the laser beam emission was started and the sample was allowed to interact with the power modulated laser beam at the first operational wavelength in the measurement range. The signal from the sample was recorded, then the laser emission was tuned to the next wavelength value. The measurement procedure was repeated for the selected wavelengths accessible to our laser, all of them contributing to realizing a single spectrum. Each sample has been measured six times. Each point in the spectra has been obtained by averaging 10 acquisitions. The LPAS system was characterized in terms of emission profile, frequency response, and signal linearity.

\subsubsection{QCL power emission profile}

Absorption data were processed to make experimental data not dependent measurement settings such as power variations, or on the amount of used sample. In this case, the absorption data have been normalized with respect to the power of the laser beam acquired for each sample measurement. A recording of the power spectrum of the radiation emitted by the laser source is reported in Figure 2.

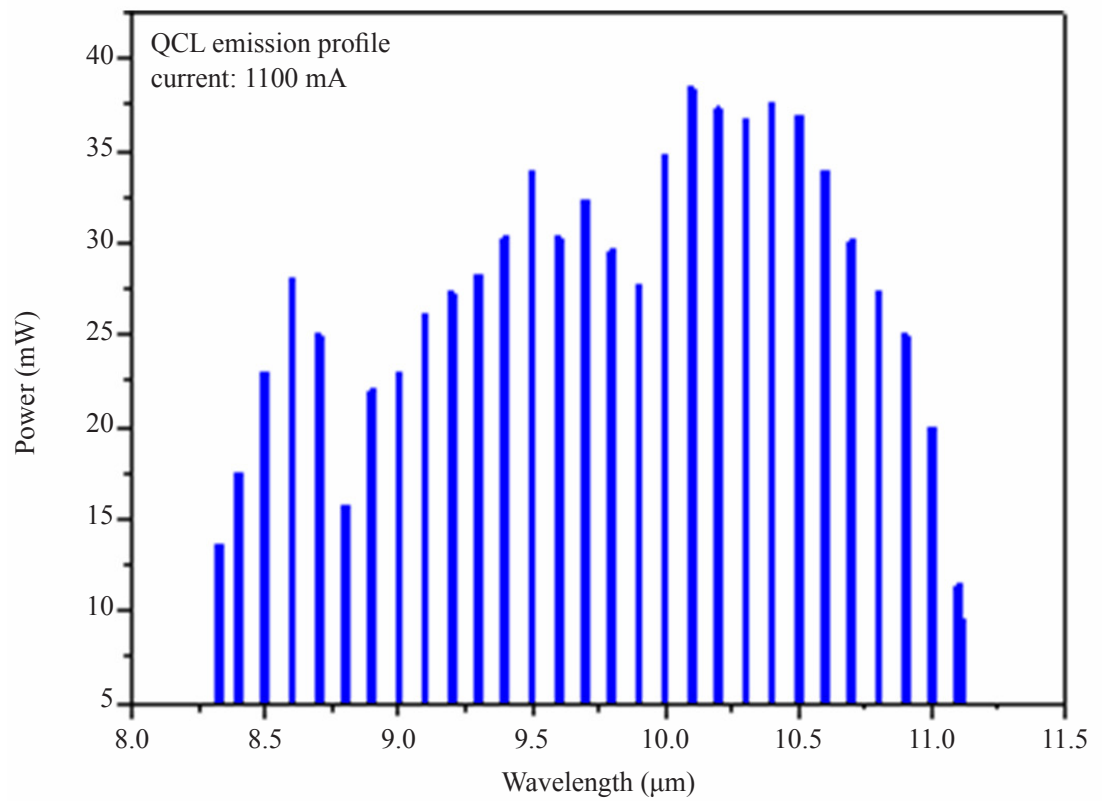

Figure 2. QCL power emission profile

\subsubsection{Cell frequency response}

The cell frequency response has been determined by measuring the PA signal (V) at a fixed wavelength of $10 \mu \mathrm{m}$ 
while varying the chopping frequency. In order to determine the resonance frequency of the PA cell with high SNR, a good absorber of IR radiation such as charcoal activated granules (Carlo Erba, Batch Number Q1D099281F) was used and a low-pass filter (model 5489A by HP) set to 10X gain has been interposed between a microphone and lockin amplifier. The empty cell and the sample of charcoal have been irradiated at $10 \mu \mathrm{m}$ where the average laser power is higher. The obtained frequency response curve is presented in Figure 3. Each point represents the average of 10 measurements. Each measurement lasts $1 \mathrm{~s}$. As expected, the response of the empty cell was almost flat and zero (not shown), while a clear resonance at $215 \mathrm{~Hz}$ can be observed with the charcoal sample.

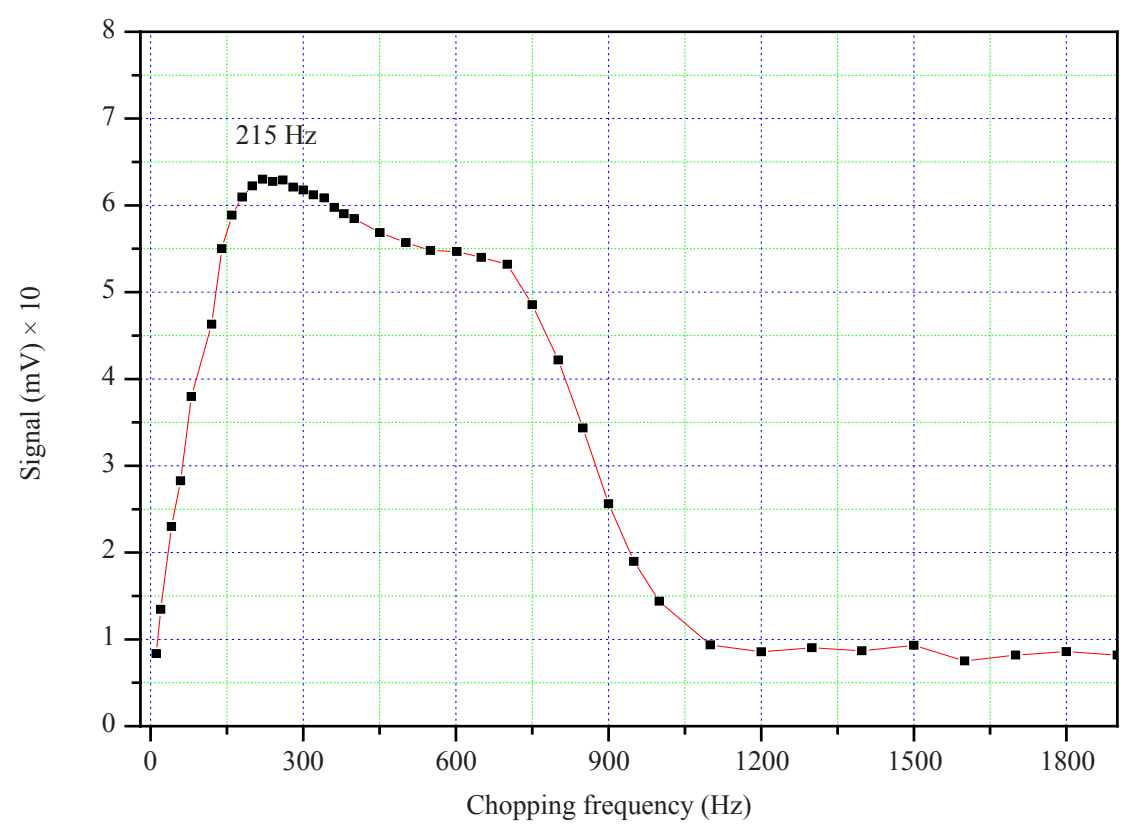

Figure 3. PA cell response frequency

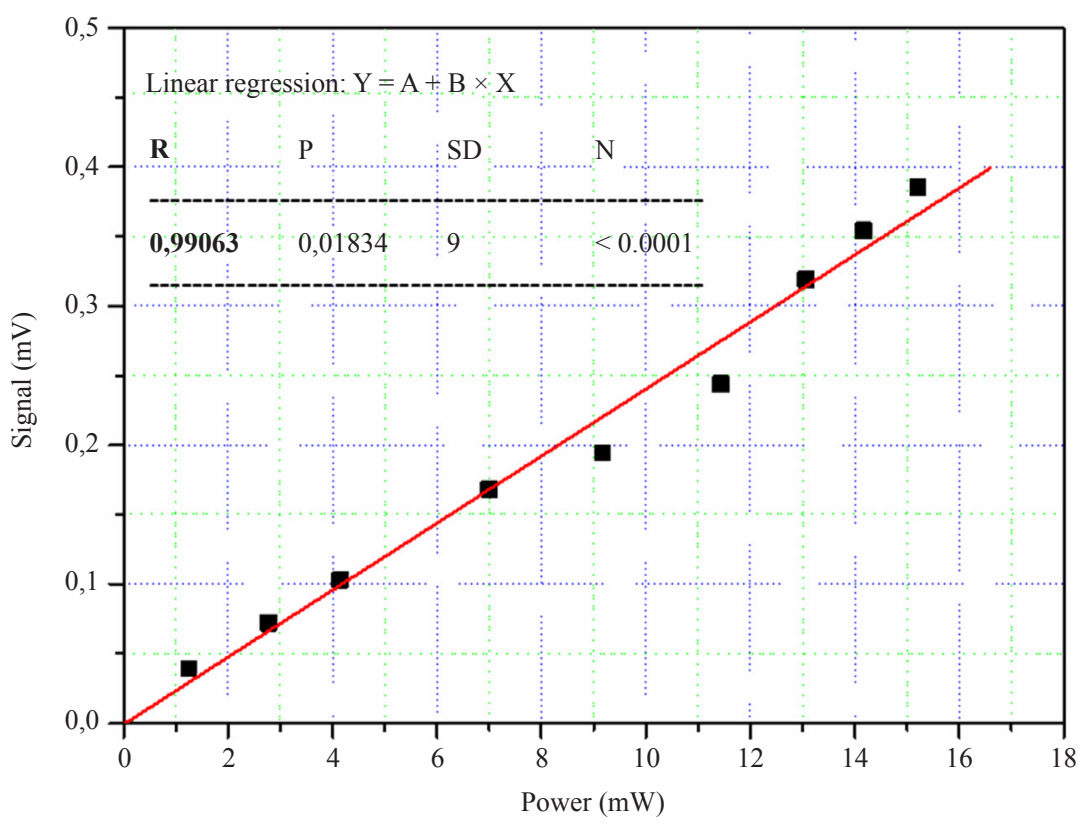

Figure 4. Linearity of the PA response vs laser power increment 


\subsubsection{Signal linearity test}

The linear increasing of the PA signal with the increasing of the laser power has been tested at $10 \mu \mathrm{m}$. Each point represents the average of 10 measurements. Each measurement lasts 1 s. As it can be noticed in Figure 4, the microphone signal is linearly increasing with the laser power, and no microphone saturation was registered in the inspected power range, although for charcoal the PA signal is maximum due to strong absorption (it is black).

\subsection{Data treatment}

Generally, a specific molecular group can be discriminated within a spectrum, but often more complex information which needs to be dealt with numerical algorithms or with complex statistical methods is requested. While the first approach tries to extract the bands/lines corresponding to tabulated values in standard databases with extraction and comparison algorithms (neural networks or similar), statistical methods (PCA-Principal Component Analysis and others) try to extract the information we need from a large number of variables in somehow related to each other. In the case of Raman, FT-IR, and LPAS spectroscopy techniques, it is possible to assign bands of vibration of some functional groups belonging to different molecules inside the sample with a good approximation, but when the spectral information is too complex to allow a rapid identification of the species in the sample, statistical analysis comes into help.

In the present work, PCA, a powerful statistical tool described for the first time in 1901 by Pearson [16], was used. PCA applies a calculation algorithm that allows reducing the size of a data set and rendering evident statistical differences among the analyzed samples. The algorithm finds a number of hypothetical variables called Principal Components (PC), representing as much as possible the variability in multivariate data [17-18]; the new variables are the result of a linear combination of the original variables, where each descriptor has its own "weight". Generally, according to a descending order of weight, the first few new variables are able to represent most of the variation in the original variables.

PCA for classification purposes was discussed in detail elsewhere [19], consequently here we only summarize the statistical procedure. As we have just said, PCA is made in order to eliminate data redundancy by changing the number of variables and consequently representing the sample set in a reduced dimension hyperspace [20]. On a data matrix containing elements $x_{i k}$, where index $k$ is used for the experimental measurements and index $i$ for the samples under study, the PCA model is described by Eq. (1):

$$
x_{i k}-\bar{x}_{k}=e_{i k}+\sum_{J=1}^{N} t_{i j} p_{j k}
$$

where the parameters $p_{j k}$ and $t_{i j}$ are calculated by minimizing the squared residuals, after subtracting $x_{k}$ the mean value of the experimental averages. In this model, the loadings $p_{j k}$ depend only on the experimentally measured variables, and the scores $t_{i j}$ only depend on the sample constituents. The number $N$ of significant components is determined by elimination and then checked by cross-validation [21].

\section{Results and discussion}

\subsection{FT-IR spectra of rice flours}

In order to identify the spectral range most useful for the investigation by LPAS method (where the differences among the samples are more evident), all the rice samples were firstly analyzed with FT-IR/ATR. Figure 5 shows the FT-IR/ATR absorption spectra of the samples under study. Different transitions of the vibrational modes can be observed in the fingerprint region between 1800 and $625 \mathrm{~cm}^{-1}(5.55-16 \mu \mathrm{m})$ where carbohydrates, proteins, and fats generally show their characteristic bands [22-25]. The results indicate that vibrational spectra of the different rice cultivars are quite similar along all spectrum, except the interval $1200-900 \mathrm{~cm}^{-1}$, where differences in intensity among samples are noticed (Figure 6). 


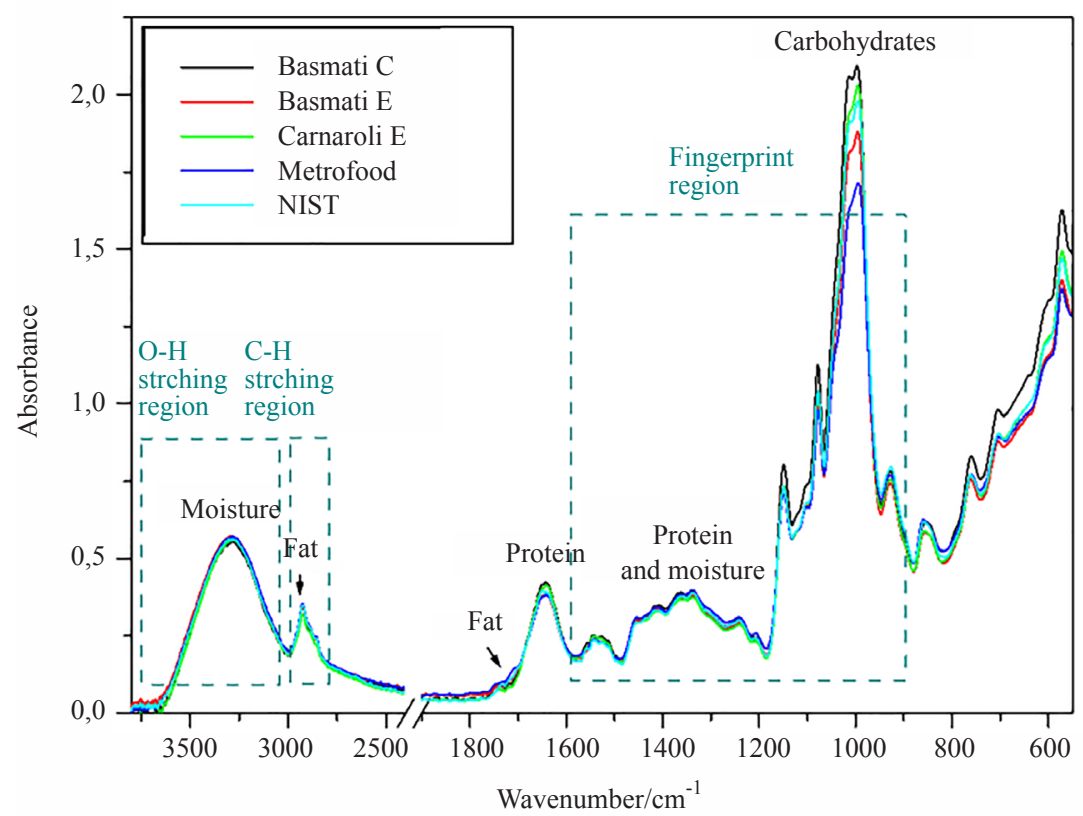

Figure 5. FT-IR spectra of different rice flours (Basmati $\mathrm{C}$ and Basmati $\mathrm{E}$ are provided by different companies; Metrofood is a certified Carnaroli cultivar; NIST is a standard flour obtained from $100 \%$ long grain river rice)

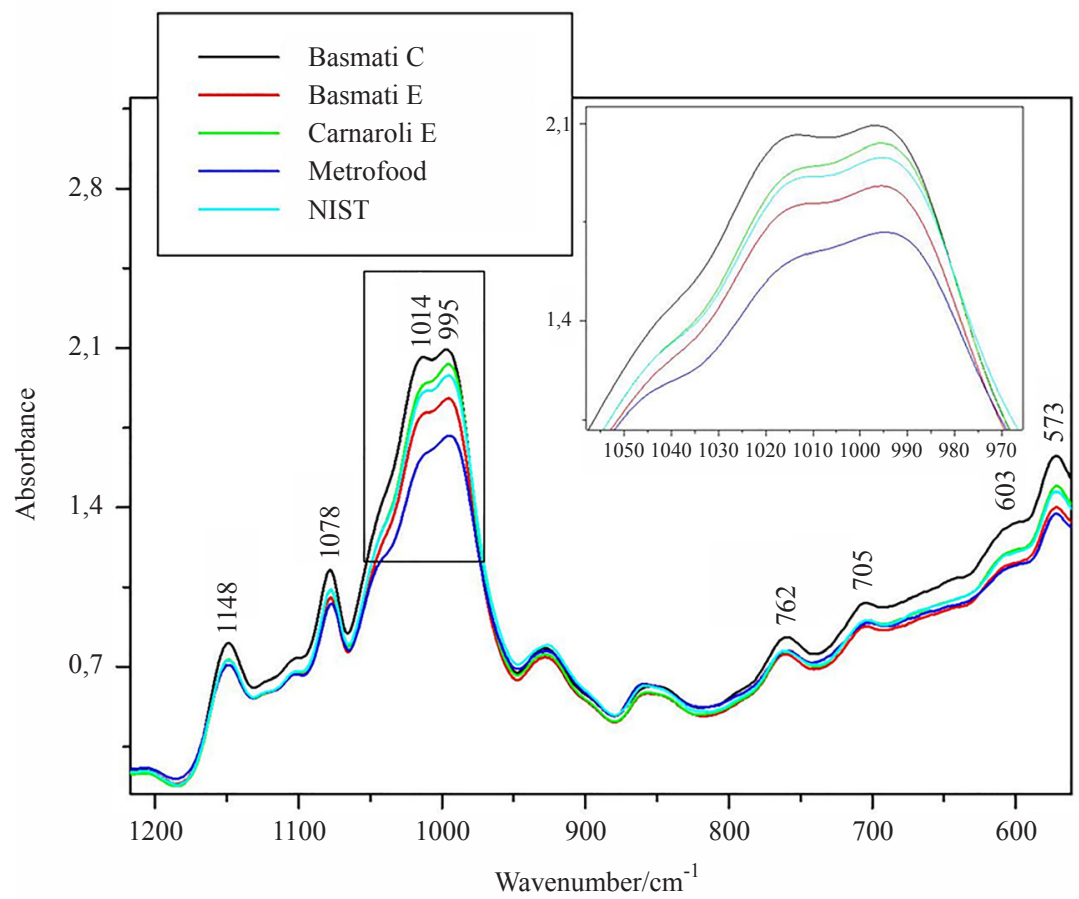

Figure 6. Zoom in the FT-IR/ATR spectra in the $1200-550 \mathrm{~cm}^{-1}$ range which put in evidence the different spectral intensities

In Figure 6, the main bands localized at 1148, 1078, 1014, $995 \mathrm{~cm}^{-1}$ followed by less significant bands at 762 , $705,603,573 \mathrm{~cm}^{-1}$ show different absorption intensities for the five rice $\mathrm{cv}$. They are mostly due to differences in the content of carbohydrates (i.e. amylose and amylopectin) and proteins (i.e. albumin and glutelin) which are present in various amounts depending on the $\mathrm{cv}$ type. For this reason, the region $1175-909 \mathrm{~cm}^{-1}(8.5-11 \mu \mathrm{m})$ was selected for the 
investigation through the LPAS system based on QCL. This range is covered by the QCL emission.

\subsection{LPAS spectra of rice flours}

Under the specified measurement conditions, measurable PA signals emitted by the rice samples were detected in the entire accessible spectral range from 8.5 to $11 \mu \mathrm{m}$. The spectral profiles of studied rice samples obtained by the LPAS technique are shown in Figure 7. Each spectrum shown in this figure represents the average of six spectra acquired for the same sample. The experimental measurement error was evaluated as the standard error of 10 acquisitions on each laser emission wavelength. The standard error was less than $2 \%$ of the measured value, which means good data reproducibility and high stability of the measurement system. As mentioned before, the absorption data were processed to make them not dependent on measurement settings, or the amount of sample used. In the specific case, the absorption data were normalized with respect to the registered laser power as well as to the sample mass unit. In Figure 7 the PA signal emitted by the empty cell (the background signal) is also shown. As it can be seen the background signal was found negligible compared to the sample signals, so it was considered to not affect the experimental spectra.

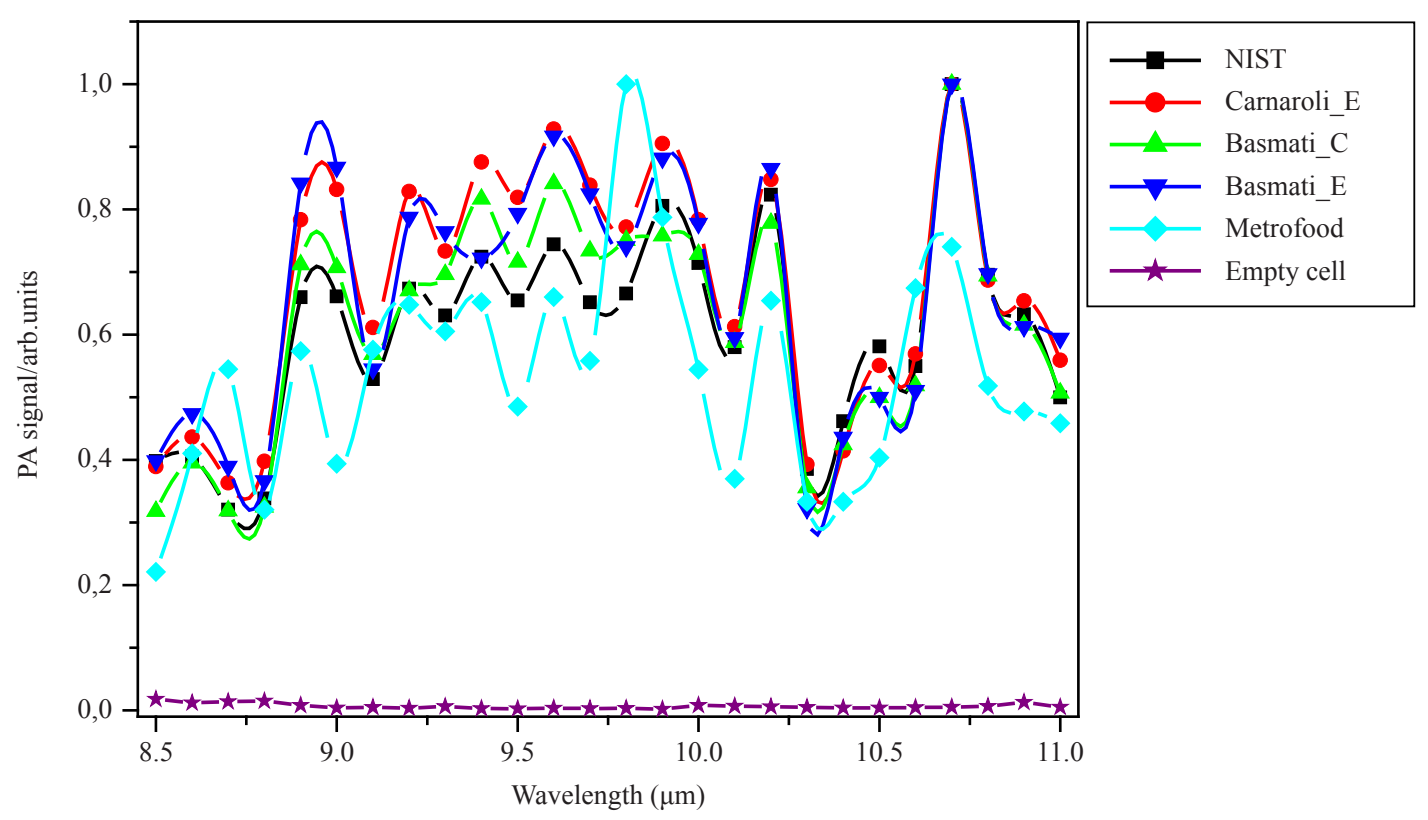

Figure 7. LPAS spectra of different rice varieties. Carnaroli, Basmati_C and Basmati_E are commercial samples and of the empty PA cell

The obtained results highlight differences in signal intensity for each examined sample. Regarding the assignment of the bands, they are in accordance with data reported in the literature [24-25].

\subsection{Principal component analyses}

When dealing with a large quantity of spectral data it is difficult to classify the samples by just looking at the LPAS spectra. Thus, the set of experimental data acquired with the LPAS system was analyzed by the PCA. Figure 13 shows that chemometric analysis turns out a successful identification method even when the differences in the spectral fingerprints are subtle. Before applying the calculation algorithm, the LPAS absorption data were normalized to their maximum peak value, in order to make them directly comparable to each other. The PCA algorithm applied to the LPAS data produced the sample distribution shown as a 2D graph (Figure 8). On each axis, the values of the two main components are reported as PC1 and PC2 which respectively account for $67 \%$ and $27 \%$ of the global variability 
of the analyzed data. Taking into account the respective contribution of each major component to the classification of substances, it resulted that the first three PCs are explaining 96\% of the overall spectral differences. Moreover, since the sum of the first two PCs exceeds $90 \%$ of the overall variability, we neglected the PC3 (2\%) and limited the PCA representation to the $2 \mathrm{D}$ classification reported in Figure 8 that highlights the five different groups corresponding to the five types of samples. The PCA analysis shown in Figure 8 emphasizes the small differences already observed among spectra of the samples in Figure 7.

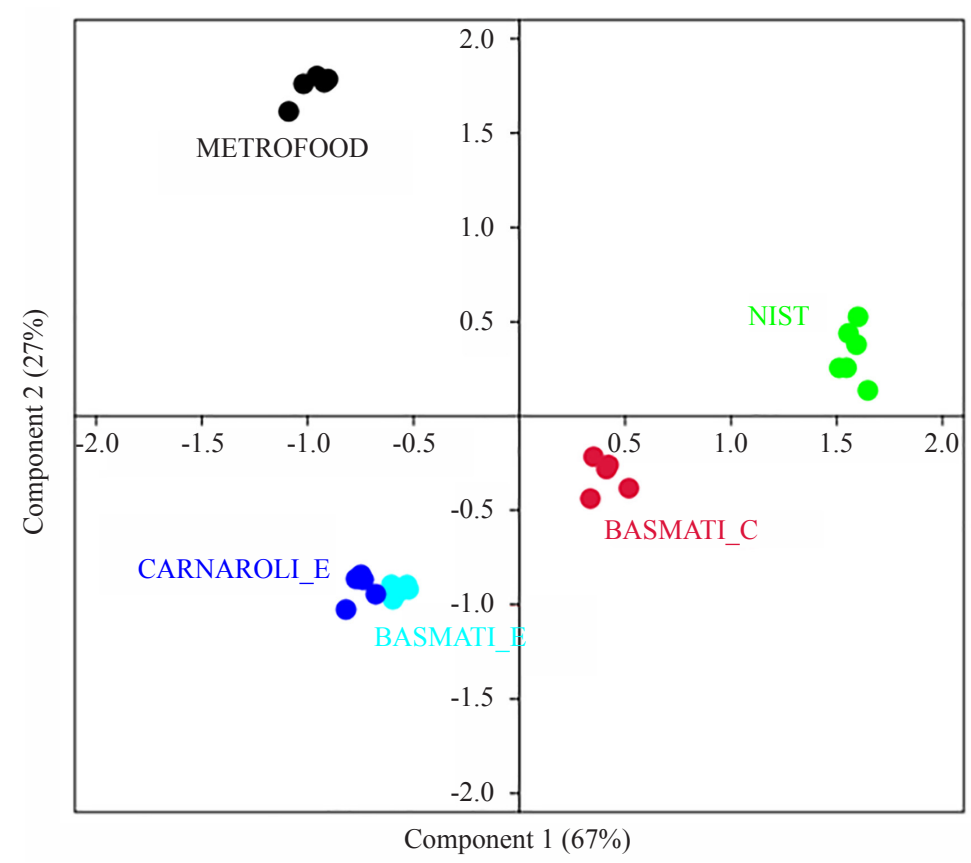

Figure 8. Result of the PCA applied to the LPAS spectra of the rice flour samples

The use of PCA appears extremely suitable for the spectroscopic analysis object of the present work. It allows to clarify the degree of similarities and/or differences between spectra, excludes overlapping of data, and highlights the most involved wavelengths in each PC (loadings). The loadings graph for the first two PCs is shown in Figure 9.

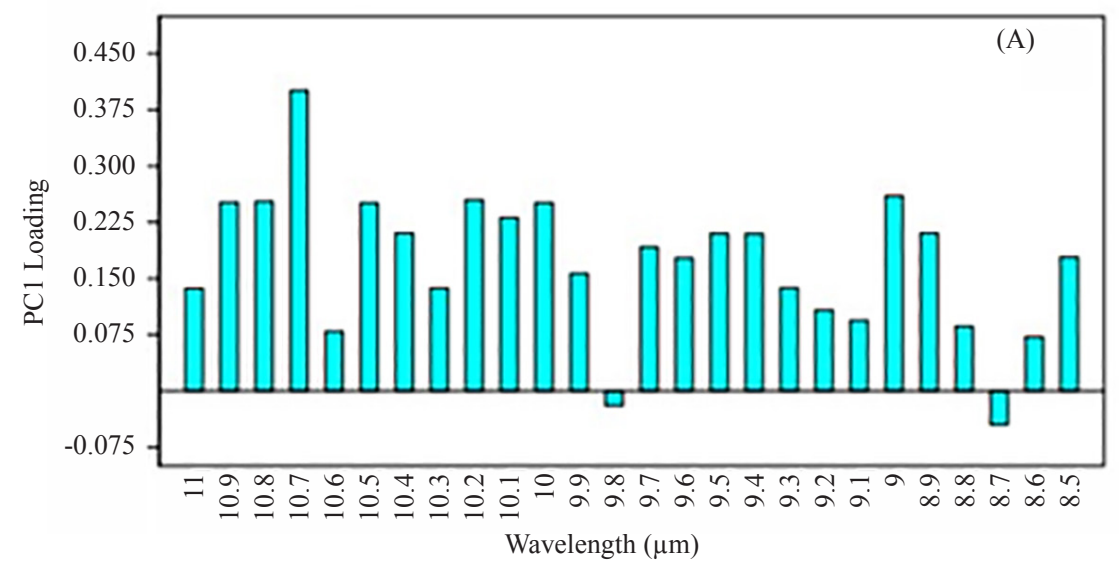




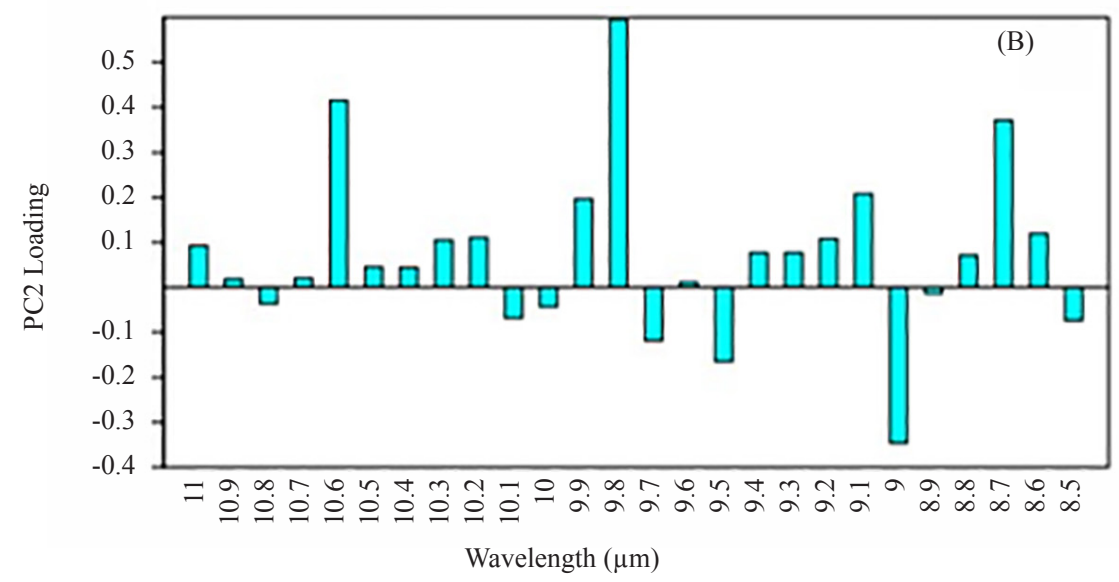

Figure 9. Loadings plot for PC1 (A) and PC2 (B)

\section{Discussion}

The LPAS frequencies were compared to the FT-IR transition frequencies. This comparison is reported in Table 1 that shows also the possible assignments of the most evident peaks of the spectra of the analyzed samples. Carbohydrates are assigned between about 9 and $10 \mu \mathrm{m}$ while proteins and the associated chemical groups are found at 8.7 and over $10.6 \mu \mathrm{m}$. In Table 1, in addition to the good agreement between FT-IR and LPAS data, we observe that the IR radiation absorptions generating LPAS spectra in the 8.5-11 $\mu \mathrm{m}$ range $\left(1175-909 \mathrm{~cm}^{-1}\right)$ are substantially ascribable to transitions between vibrational modes of the $\mathrm{S}=\mathrm{O}, \mathrm{C}-\mathrm{O}$ and $\mathrm{C}-\mathrm{C}$ bonds.

Table 1. Frequencies of the IR transitions compared with LPAS transitions for rice and their suggested assignments [24-25]. In the two panels fall the frequencies of transitions in the range of interest $8.5-11 \mu \mathrm{m}\left(1175-909 \mathrm{~cm}^{-1}\right)$

\begin{tabular}{ccc}
\hline FTIR peak position $\left(\mathrm{cm}^{-1}\right)$ & LPAS peak position $\left(\mathrm{cm}^{-1}\right)$ & Assignment \\
\hline 1150 & 1150 & $\mathrm{~S}=\mathrm{O}$ (in albumin, globulin and glutelin) \\
1078 & 1078 & C-O (in albumin, globulin and glutelin) \\
1028 & 1033 & C-O (in albumin, globulin and glutelin) \\
927 & 930 & C-C (in albumin, globulin and glutelin) \\
\hline
\end{tabular}

Other spectral features not indicated in the table could be assigned to other components present in the rice composition such as carbohydrates, fat, vitamins, etc. In fact, from the nutritional point of view, rice is high in starchy carbohydrates and low in fat, is packed full of minerals and provides an excellent source of B, E, and other vitamins (thiamin, niacin, and riboflavin), Zinc, Potassium, etc.

The experimental LPAS spectra are rich in spectral features. Nevertheless, a group classification of the samples cannot be easily performed if only based on the direct comparison among the LPAS spectra. For this reason, a PCA statistical approach to the LPAS data collection was required. On the PCA graph (see Figure 8), the analyzed varieties of rice flour appear well separated and without overlapping areas, with exception of Basmati_E. In fact, Basmati_E is clustering very close to Carnaroly_E, suggesting that inside this sample, a high percentage of Carnaroli cv could have been added. The Metrofood sample separates distinctly from both the Carnaroli_E, Basmati_E, Basmati_C, and the NIST standard, suggesting a different distribution and chemical concentration of the metabolites. Since Carnaroli_E is clustering far from Metrofood (cv Carnaroli) it can be assumed that Carnaroli_E could be aged, but more experiments are needed to confirm this hypothesis. 
The loadings plot indicates that $10.7 \mu \mathrm{m}, 10.6 \mu \mathrm{m}, 9.8 \mu \mathrm{m}$, and $9.0 \mu \mathrm{m}$ are the most useful wavelengths for the discrimination of the samples. This information turn-out is useful in the view of building up a simplified and very cheap LPAS prototype that used only these wavelengths to compute the specific sample identification task in a few seconds.

We emphasize that from the bibliographic research, however, no work has emerged that reported high resolution rice spectroscopy studies, in particular, no studies have emerged concerning the application of the LPAS technique to rice flours.

\section{Conclusions}

QCL-LPAS instrument has been characterized by determining the frequency response of the PA cell, the signal linearity, and the laser emission profile. Based on this, the conditions for optimum operation have been settled. The comparison between the NIST standard, Carnaroli, and Basmati commercial samples, and the Metrofood highlighted similar spectral profiles. Beyond the theoretical interpretations of spectroscopic data, the LPAS spectra put in evidence that each substance shows a characteristic spectral pattern, also appreciable by a subjective visual evaluation that considers the overall trend of the graphs. Nonetheless, a direct comparison between individual spectra is difficult to quantify. For this reason, a statistical treatment based on the PCA has been applied to the set of spectral data, in order to demonstrate in an incontrovertible way the ability of the system to distinguish between different varieties of rice that cluster clearly. The statistical analysis allowed to classify samples of different varieties in groups.

In conclusion, this study proved that LPAS techniques coupled with PCA was able to distinguish different rice flours because of their different metabolic content. Due to its versatility, such a method could be proposed also to monitor the aging or shelf life of rice flour as well as rice grains or other agro-food products such as fruits and vegetables. The adulteration is rife in almost all agricultural food products. Since distinguishing the adulterant with look-alike food products is difficult with naked eye visual observation, LPAS could be applied as a fast and nondestructive method to detect any kind of food adulteration [26-28].

\section{Acknowledgements}

The support of SAL@CQO Project (Italian Ministry of Economic Development, Programma Industria 2015) and SafeFood Workpackage of TechHea Project (ENEA) is gratefully acknowledged. A rice sample has been provided by the PRO-METROFOOD Project which has received funding from the European Union's Horizon 2020 research and innovation programme under grant agreement No 739568. The authors are grateful to Roberta Fantoni and Antonio Palucci for constant encouragement.

\section{Conflict of interest}

The authors declare that there are no conflicts of interest regarding the publication of this article.

\section{References}

[1] Giovenzana V, Beghi R, Civelli R, Guidetti R. Optical techniques for rapid quality monitoring along minimally processed fruit and vegetable chain. Trends in Food Science \& Technology. 2015; 46: 331-338.

[2] Costa G, Bonora E, Fiori G, Noferini M. Innovative non-destructive device for fruit quality assessments. Acta Horticulturae. 2011; 913: 575-581.

[3] Merzlyak MN, Gitelson AA, Chivkunova OB, Rakitin VY. Non-destructive optical detection of leaf senescence and fruit ripening. Physiologia Plantarum. 1999; 106(1): 135-141.

[4] Moore JC. Food fraud: public health threats and the need for new analytical detection approaches. Cornell University eCommons. Available from: https://core.ac.uk/reader/83605803 [Accessed 2nd February 2021]. 
[5] Giubileo G, Lai A, Piccinelli D, Puiu A. Laser diagnostic technology for early detection of pathogen infestation in orange fruits. Nuclear Instruments and Methods in Physics Research A. 2010; 623: 778-781.

[6] Giubileo G, Calderari I, Puiu A. High resolution spectroscopy of sweeteners. Proceedings of the 3rd International Conference on Photonics, Optics and Laser Technology. 2015; 1: 91-95. Available from: DOI: 10.5220/0005336600910095.

[7] Giubileo G, Puiu A, Nunziante CS. Spettroscopia della melammina con laser a $\mathrm{CO}_{2}$. Technical Reports of the Italian National Agency for New Technologies, Energy and Sustainable Economic Development. RT/2016/23/ ENEA. 2016.

[8] Fiorani L, Giubileo G, Mangione L, Puiu A, Saleh W. Food fraud detection by laser photoacoustic spectroscopy. Technical Reports of the Italian National Agency for New Technologies, Energy and Sustainable Economic Development. RT/2017/41/ENEA. 2017.

[9] National Institute of Standards and Technology, National Institute of Standards \& Technology Certificate of Analysis Standard Reference Material 1568b. Available from: https://www-s.nist.gov/srmors/certificates/1568b.pdf [Accessed 5th November 2018].

[10] The Food and Agriculture Organization of the United Nations. Rice consumption in major rice consuming countries. Available from: http://www.fao.org/3/Y6618E/Y6618E00.htm\#nutri [Accessed 22nd August 2021].

[11] Kraehmer H, Thomas C, Vidotto F. Rice production in Europe. In: Chauhan B, Jabran K, Mahajan G. (eds.) Rice Production Worldwide. Switzerland: Springer; 2017.

[12] European Commission, Food, Farming, Fisheries. Available from: http://www.fao.org/3/Y6618E/Y6618E00. htm\#nutri [Accessed 22nd August 2021].

[13] Vemireddy LR, Satyavathi VV, Siddiq EA, Nagaraju J. Review of methods for the detection and quantification of adulteration of rice: Basmati as a case study. Journal of Food Science and Technology. 2015; 52: 3187-3202.

[14] Tomas EF. Infrastructure for promoting metrology in food and nutrition. Food Quality and Technology. Institute of Agrifood Research and Technology; 2019. Available from: http://www.metrofood.eu/ [Accessed 21st December 2020].

[15] Haisch C. Photoacoustic spectroscopy for analytical measurements. Measurement Science and Technology. 2012; 23: $12001-12018$.

[16] Pearson K. On lines and planes of closest fit to systems of points in space. Philosophical Magazine. 1901; 2: 559-572.

[17] Davis JC. Statistics and Data Analysis in Geology. USA: John Wiley and Sons; 1986.

[18] Harper DAT. Numerical Palaeobiology. USA: John Wiley and Sons; 1999.

[19] Brown SD, Tauler R, Walczak B. Comprehensive chemometrics, chemical and biochemical data analysis. Oxford: Elsevier; 2009.

[20] Giubileo G, Colao F, Puiu A. Identification of standard explosive traces by infrared laser spectroscopy: PCA on LPAS data. Laser Physics. 2012; 22(6): 1033-1037. Available from: https://doi.org/10.1134/S1054660X12060035.

[21] Diana G, Tommasi C. Cross-validation methods in principal component analysis: A comparison. Statistical Methods \& Applications. 2002; 11: 71-82.

[22] Sujka K, Koczon P, Ceglinska A, Reder M, Ciemniewska-Zytkiewicz H. The application of FTIR spectroscopy for quality control of flours obtained from polish producers. Journal of Analytical Methods in Chemisrty. 2017; 2017: 1-9. Available from: https://doi.org/10.1155/2017/4315678.

[23] Genkawa T, Ahamed T, Noguchi R, Takigawa T, Ozaki Y. Simple and rapid determination of free fatty acids in brown rice by FTIR spectroscopy in conjunction with a second-derivative treatment. Food Chemistry. 2016; 191: 7-11.

[24] Farooq Z, Ismail AA. QA/QC of flours using the agilent cary 630 ATR-FTIR analyzer. Application NoteFood Testing and Agriculture. Available from: https://www.agilent.com/cs/library/applications/5991-0785EN_ AppNote_630_Flour.pdf [Accessed 8th Jenuary 2021].

[25] Guo Y, Cai W, Tu K, Tu S, Wang S, Zhu X, et al. Infrared and Raman spectroscopic characterization of structural changes in albumin, globulin, glutelin and prolamin during rice aging. Journal of Agricultural and Food Chemistry. 2013; 61: 185-192.

[26] Reis N, Franca AS, Oliveira LS. Quantitative evaluation of multiple adulterants in roasted coffee by Diffuse Reflectance Infrared Fourier Transform Spectroscopy (DRIFTS) and chemometrics. Talanta. 2013; 115: 563-568.

[27] Fiorani L, Artuso F, Giardina I, Lai A, Mannori S, Puiu A. Photoacoustic laser system for food fraud detection. Sensors. 2021; 21: 4178. Available from: https://doi.org/10.3390/s21124178.

[28] Galvin-King P, Haughey SA, Elliott CT. Herb and spice fraud; the drivers, challenges and detection. Food Control. 2018; 88: 85-97. 